\title{
A Gait Analysis Study on Charcot-Marie-Tooth Patients
}

\author{
A. Mehrpouya ${ }^{1}$, M. Haddadi Dashtbayaz ${ }^{1}$, A. Zarif Allali ${ }^{1}$, A. Ashofteh Yazdi ${ }^{1, *}$, R. Boostani ${ }^{2}$ \\ ${ }^{1}$ Department of Biomechanics, Faculty of Engineering, Mashhad Branch, Islamic Azad University, Mashhad, Razavi Khorasan \\ province, Iran. \\ ${ }^{2}$ Department of Neurology, Mashhad University of Medical Sciences, Mashhad, Razavi Khorasan province, Iran.
}

How to cite this paper: A. Mehrpouya, M. Haddadi Dashtbayaz, A. Zarif Allali, A. Ashofteh Yazdi, R. Boostani. (2022) A Gait Analysis Study on Charcot -Marie-Tooth Patients. International Journal of Clinical and Experimental Medicine Research, 6(1), 74-80. DOI: $10.26855 /$ ijcemr.2022.01.013

Received: November 15, 2021

Accepted: December 18, 2021

Published: January 18, 2022

*Corresponding author: A. Ashofteh Yazdi, Department of Biomechanics, Faculty of Engineering, Mashhad Branch, Islamic Azad University, Mashhad, Razavi Khorasan province, Iran.

Email: a.ashofte@srbiau.ac.ir

\begin{abstract}
The aim of this study was to quantitatively investigate the kinematics and kinetics characteristics of Charcot-Marie-Tooth patients, in order to extract motion model parameters and provide objective diagnostic criteria. CharcotMarie-Tooth (CMT) is the most common disease among all neuropathies of the peripheral nervous system and leading to disturbances in walking and balance in the person. For this purpose, the motion analysis approach was performed on a group of patients with CMT $(\mathrm{N}=5)$ and a control (healthy) group $(\mathrm{N}=5)$. By recording the spatial and temporal parameters of participant's joints with QTM software, the appropriate non-linear models of motion were extracted. To the purpose of providing CMT diagnostic criteria, the coefficients of kinematic and kinematic model parameters were compared for the two sample groups. The results showed the highest decrease in the range of motion and angular velocity of the knee and ankle joints from healthy to patient samples, while no significant changes were observed for the hip joint. The changes in motion model parameters were mostly observed in directions $\mathrm{y}$ and $\mathrm{x}$, while the maximum peripheral kinetic parameters were measured in the fore-heel for the patients compared to the healthy group.
\end{abstract}

\section{Keywords}

Biomechanics, Motion Analysis, Diagnostic Criteria, Genetic Charcot-Marietooth

\section{Introduction}

CMT is a neurological and genetic-environmental heterogeneous disorder that affects one person in each 2,500 individuals. According to this statistic, it afflicts 125,000 individuals in the United States [1]. CMT is the most common disease among all neuropathically disorders of the environmental nervous system [2,3].

CMT is generated by mutations that cause defects in nerve proteins. Neural signals are produced by an axon with a myelin sheath wrapped around it. Most of the CMT mutations affect the myelin sheath, but some affect axon. The myelin sheath allows a faster signal transmission to neurons. When the myelin sheath is damaged, the nerve signal becomes slower; the electromyography or EMG can evaluate this procedure. However, a damaged axon reduces the potential for muscle activity. Jean-Martin Charcot (1825-1893), his pupil Pierre Marie (1940-1853) and Howard Henry Tooth, recognized this disease therefore; it is named after them [4, 5, 6].

CMT signs usually begin in childhood or adolescence, but some people do not experience symptoms until the age of 30 or 40. The first symptoms of CTM usually include disturbances in balance and carelessness. Patients af- 
flicted with CMT may experience abnormalities in the leg or hands such as curvature of the foot or atrophy of the hands [7]. These individuals usually have difficulty in bending and twisting the foot or walking on the heel. These disorders may generate taller footsteps and may increase the risk of ankle injury and stumbling [8].

Symptoms and progression of CMT may vary in individuals; these include unwanted damages to the teeth, respiratory problems, and digestive system [9]. Majority of these patients often occupy more time in the localized phase during walking [10]. Increased Single support also plays a major in the movement of CMT patients. Following symptoms are abundantly found in these individuals: small steps, the increased height of the feet during walking, the limitation of dorsiflexion and plantarflexion and the reduction of muscle strength regarding the dorsiflexors and pluton flexors [11, 12].

The biomechanical examination of motion in the detection lesions and gait diseases is very practical. The biomechanical analysis of motion involves the study of kinematic and kinetic parameters in a static or dynamic state. With the help of kinematic parameters (velocity, acceleration, displacement) and kinetic parameters (force, pressure, torque), models of motion can be obtained [13, 14, 15].

The kinematics and kinetics motion analysis of patients with peripheral neuropathy have been done in the recent years in a variety of ways. A recent study was conducted on a large number of patients with peripheral neuropath investigating the kinetic and kinematic parameters of the patients [16]. A gait analysis study was also performed on 25 subjects with peripheral neuropathy and 27 healthy subjects [17]. A decrease in the range of motion in the ankle joint of patients during flexion and extension was generally observed. The duration of support and the length of the gate cycle were seen to be higher in patients than healthy group. Foot pressure was found to increase in patients with neuropathy.

The aim of this study was to quantitatively investigate the kinematic and kinetic parameters of Charcot Marie Tooth patients, in order to extract a motion model and provide standard criteria for diagnosis of the disease. Comparison of gait model parameters of patients with healthy group can determine the difference in the range of motion and can improve the diagnosis of motor disabilities.

\section{Materials and Methods}

\subsection{Sampling}

The procedures conform to the international and institutional guidelines for the use of human in the experimentations. An informed consent was obtained for experimentations from all the participants. Motion analysis tests were performed on two groups of 5 samples; CMT patients and a control (healthy) group. Only five individuals' patients were selected for CMT motor testing due to the same progressed degree of the disease. The entire patients suffered from neuropathy due to CMT and had no secondary complications such as lower limb operations, bone fracture or joint damage. A group of control (healthy) volunteers also participated in the motion tests. Age, height and weight and statistical population of the participants were also controlled (Table 1).

Table 1. Baseline subjects' characteristics for the two groups

\begin{tabular}{ccc}
\hline & Patients $(\mathrm{N}=5)$ & Healthy Individuals $(\mathrm{N}=5)$ \\
\hline Sex ratio (male / female) & $5 / 0$ & $5 / 0$ \\
Avg. age & 43.4 & 23 \\
Avg. height & 184.4 & 180.8 \\
Avg. weight & 86.7 & 73 \\
\hline
\end{tabular}

\subsection{Laboratory Experiments}

The lab was equipped with eight Q5+ 200Hz cameras with $1 \mathrm{~mm}$ precision and 4 mega pixel picture quality in this lab. All the equipments were fully calibrated prior to beginning the motion tests. The movement course was considered about 10 meters that was covered with tatami flooring. Also, two force plates were in the middle of the course. The kinetic and kinematic characteristics of the individuals were recorded simultaneously with the cameras. The course length and test conditions considered identical for the patients and control group. We utilized some sort of special clothing for the movement test (Figure 1). These fabrics were designed to be tight and able to provide perfect marker stability when placing markers on joints, since movement of markers during the test augments the 
probability of noise and error in the results. We used fourteen-point markers and installed them on the ankle, knee, shoulder, hip, heel, forehead and seventh neck joint for starting the test (Figure 2).

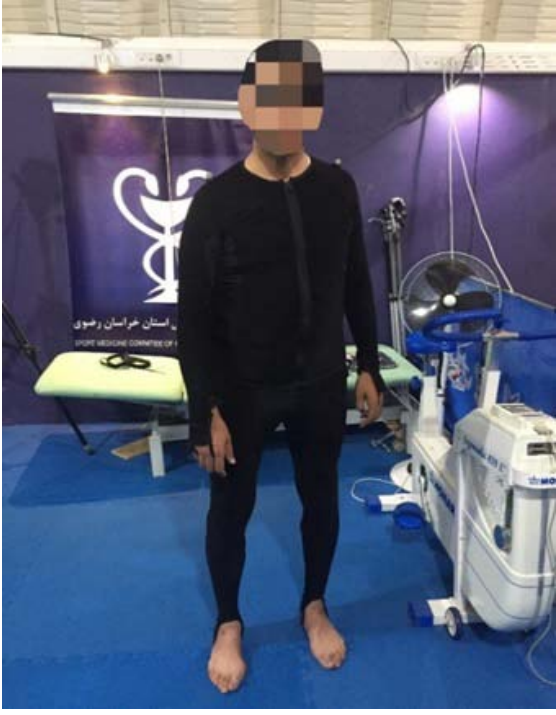

Figure 1. MOCAP clothes used in the motion test.

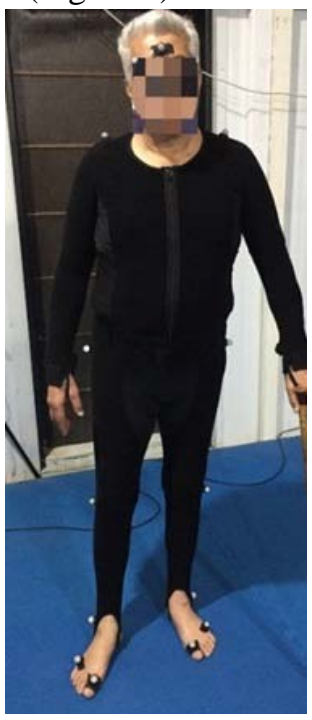

Figure 2. A view of marker placement on the joints of the body.

The gait speed was equivalent to the average speed of their daily routine as well. By applying a particular speed for a motion test, the motion pattern of patients would be altered unintentionally [18]. Each participant moved through the entire path for five times. After finishing the tests, the best cycle was selected for each sample. The tests were recorded by eight synchronized cameras which were placed at the corners of the lab (Figure 3). Camera positions were intended to monitor and cover all the anatomical plates of the person while on the move.

The position-time and force-time data of each marker were accurately recorded at any moment along the course of examination. The cameras captured kinematic characteristics by recording momentarily the position of the joints during the movement of individuals. The force plates also recorded kinetic characteristics simultaneously by recording the strength of soles. All kinetic and kinematic characteristics were recorded in three $\mathrm{x}, \mathrm{y}$ and $\mathrm{z}$ directions.

The motion characteristics were recorded by simulating joints using the Qualisys Tracker Manager (QTM) software. In this software, the positive axis of $X$ marked the forward movement, the positive axis of $Y$ marked the medial transverse direction and the positive axis of $\mathrm{Z}$ marked the upward movement and perpendicular to the ground (Figure 4). At some points during the examination, the markers were hidden from the camera's viewpoint and the characteristics were not recorded in the needed fraction of the time; therefore, after accomplishment of test and registering the characteristics, a health ensuring process was applied to them. As a result, among all five examinations performed for each individual, one of them was selected as the healthiest sample.

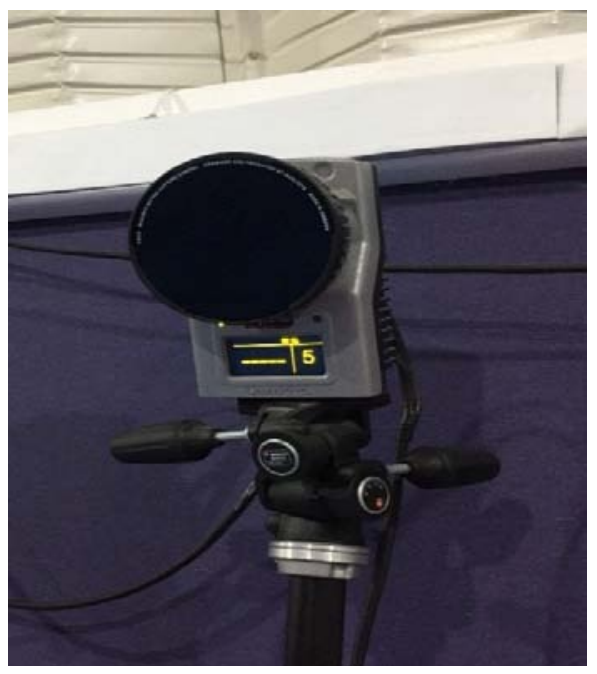

Figure 3. An illustration of Q5+ Camera used in the lab. 


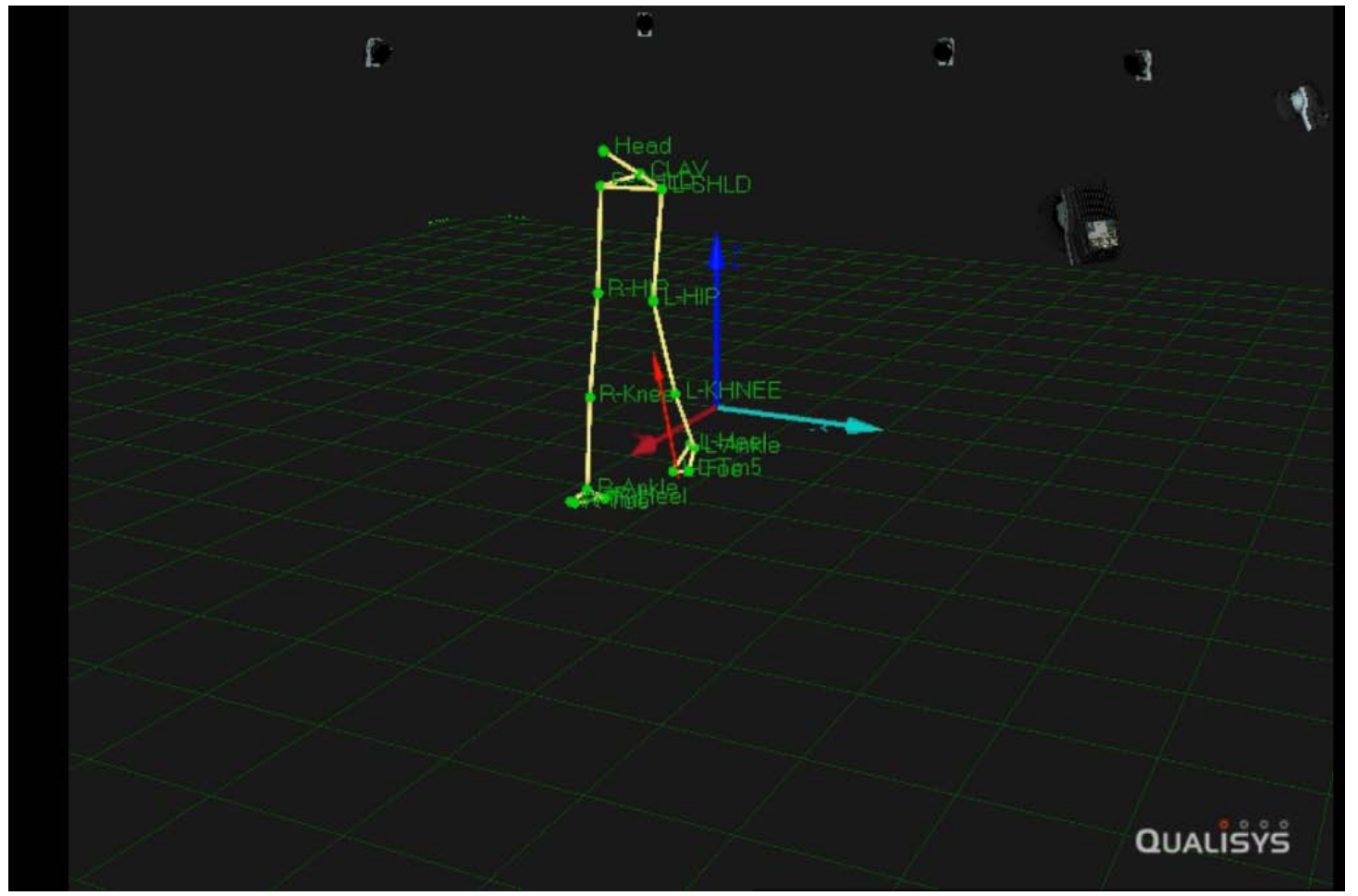

Figure 4. Joints Stimulation in QTM software.

\subsection{Data Processing}

The data was imported into Excel 2016 software. The Excel interface enabled us to monitor the data concerning each joint in separate time unit. Therefore, the best possible gait cycle of each participant was selected for further study. Data was exported to the MATLAB R2018a software environment to achieve descriptive models for the motion of individuals.

Using MATLAB software, the appropriate non-linear models fitted to the motion patterns accordingly. By assigning spatial-temporal variables of each of the joints separately, the proper functions for defining the motion models were extracted. Increasing the degree of functions led to enhanced accuracy of the fits. Fourier and Gaussian functions were selected as the best models for defining kinematic and kinetic characteristics, respectively (Equations 1 and 2).

$$
\begin{gathered}
\mathrm{Y}=\mathrm{a}_{0}+\mathrm{a}_{1} * \cos (\mathrm{x} * \mathrm{w})+\mathrm{b}_{1} * \sin (\mathrm{x} * \mathrm{w}) \\
\mathrm{Y}=\mathrm{a}_{1} * \exp -\left(\left(\mathrm{x}-\mathrm{b}_{1}\right) / \mathrm{c}_{1}\right)^{2}
\end{gathered}
$$

Three main coefficients in the Fourier functions including $w$ (angular velocity), $a_{1}$ and $b_{1}$ (weight coefficients) were considered for kinematic analysis in both control and patient groups. Similarly, Gaussian functions coefficients $\mathrm{a}_{1}$ (range of Gaussian function), $\mathrm{b}_{1}$ (center location peak) and $\mathrm{c}_{1}$ (standard deviation) considered for kinetic analysis.

A coefficient of determination $\mathrm{R}^{2}$ was considered for the acceptance of the models fitted. It modifies the ability of the models to predict the experimental results. The minimum $\mathrm{R}^{2}=0.9$ was considered for the acceptance of the models fitted to the two sample groups. Independent sample T-test, using SPSS statistics 18 software, was implemented to study the significance of the model parameters for the kinematics and kinetics changes between two sample groups. A P-value of less than 0.05 was considered as significant statistical difference [19, 20, 21].

\section{Results}

In following tables, standard deviations of the kinematic and kinetic characteristics as the mean coefficients and standard deviations of each significant model parameters for both patients and the control group were presented. The results of significant statistical differences between the angular velocity of the two sample groups in the specified markers were presented in three directions $\mathrm{x}, \mathrm{y}$ and $\mathrm{z}$ (Table 2). 
The results of significant statistical differences between the kinetic characteristics (coefficient $\mathrm{a}_{1}$ ) of the two sample groups in the specified markers were presented in three directions $\mathrm{x}, \mathrm{y}$ and $\mathrm{z}$ (Table 3 ).

The results of significant statistical differences between the kinetic characteristics (coefficient $b_{1}$ ) of the two sample groups in the specified markers were presented in three directions $\mathrm{x}, \mathrm{y}$ and $\mathrm{z}$ (Table 4).

The results of significant statistical differences between the kinetic characteristics (coefficient $c_{1}$ ) of the two sample groups in the specified markers were presented in three directions $\mathrm{x}, \mathrm{y}$ and $\mathrm{z}$ (Table 5).

Table 2. The statistical results of the angular velocity (w)

\begin{tabular}{cccccc}
\hline Joints Characteristics & Control group Mean parameters & Patient group Mean parameters & Control SD & Patients SD & P-value \\
\hline Left Ankle (x) & 1.0807 & 0.3136 & 0.51 & 0.2 & 0.007 \\
Right Ankle (x) & 1.1006 & 0.4316 & 0.5 & 0.27 & 0.015 \\
Left Knee (x) & 1.1342 & 0.6574 & 0.39 & 0.42 & 0.05 \\
Right Knee (x) & 1.0454 & 0.5938 & 0.27 & 0.42 & 0.04 \\
Right Foot Thumb (x) & 1.1081 & 0.3313 & 0.56 & 0.22 & 0.01 \\
Left Knee (y) & 1.3530 & 0.7856 & 0.47 & 0.38 & 0.02 \\
Right Knee (y) & 1.5132 & 0.8615 & 0.25 & 0.46 & 0.01 \\
Left Ankle (y) & 1.4020 & 0.6961 & 0.43 & 0.31 & 0.01 \\
Left Heel (y) & 1.4382 & 0.7016 & 0.5 & 0.38 & 0.01 \\
Right Heel (y) & 1.3765 & 0.8801 & 0.43 & 0.31 & 0.03 \\
Left Foot Thumb (y) & 1.4326 & 0.7391 & 0.47 & 0.4 & 0.01 \\
\hline
\end{tabular}

Table 3. The Statistical Results of Coefficient $a_{1}$

\begin{tabular}{|c|c|c|c|c|c|}
\hline Joints & Control group Mean parameters & Patient group Mean parameters & Control SD & Patients SD & P-value \\
\hline Right Heel (y) & 14.23 & -55.7 & 33.04 & 67.14 & 0.03 \\
\hline Left Foot Thumb (z) & -12.38 & 6.10 & 11.67 & 17.56 & 0.04 \\
\hline
\end{tabular}

Table 4. The Statistical Results of Coefficient $b_{1}$

\begin{tabular}{|c|c|c|c|c|c|}
\hline Joints & Control group Mean parameters & Patient group Mean parameters & Control SD & Patients SD & P-value \\
\hline Left Foot Force (x) & 3.1224 & 6.1150 & 1.58 & 3.37 & 0.05 \\
\hline Right Foot Force (x) & 2.6879 & 5.3114 & 1.55 & 2.55 & 0.04 \\
\hline Left Foot Force (y) & 3.0313 & 6.4186 & 1.62 & 3.38 & 0.03 \\
\hline Right Foot Force (y) & 2.9404 & 5.5176 & 1.63 & 2.66 & 0.05 \\
\hline Left Foot Force (z) & 2.6753 & 6.2946 & 2.04 & 3.41 & 0.03 \\
\hline
\end{tabular}

Table 5. The Statistical Results of Coefficient $c_{1}$

\begin{tabular}{|c|c|c|c|c|c|}
\hline Joint Characteristics & Control group Mean parameters & Patient group Mean parameters & Control SD & Patients SD & P-value \\
\hline Left sole Force $(\mathrm{x})$ & 0.1019 & 0.0409 & 0.053 & 0.034 & 0.02 \\
\hline Right sole Force $(\mathrm{x})$ & 0.0589 & 0.0284 & 0.033 & 0.014 & 0.04 \\
\hline
\end{tabular}

\section{Discussion}

Although treadmill was not used in this study, the results were comparable to the results of the same work [16, 17]. Increasing duration of the gate cycle and support in patients were similar to previous studies. Also, a decrease 
in the range of motion of ankle joint in patients with neuropathy was observed. However, no increase in foot pressure was seen in patients.

We identified the motor constraints for the CMT group with a generalized summary of the presented tables and outcomes. The $a_{1}$ coefficient representing the kinematics motion range of joints, had a significant variation in the right heel and left foot thumb. The progressive considerable enhancement of $\mathrm{a}_{1}$ coefficient of heel from control group to the patients indicated the growth of range of motion in the CMT patients. Vibrations in the lower limbs and organs adjacent to ground might led to distortion of the heel in the lateral direction of CMT patients.

The increased range of motion in the $\mathrm{z}$ axis was significant in the feet thumb. The negative mean value in the control group was due to pressing the feet thumb to the ground while taking steps. The positive sign of the average of $a_{1}$ coefficient represented an increased upward motion of the thumb in the patients group. This phenomenon was caused by sudden thumb jerks in the patients. The increased SD values of $\mathrm{a}_{1}$ coefficient in thumb and heel joints showed the data dispersion in patients due to abnormal steps.

The angular velocity was observed to have the most significant changes for CMT patients in direction $\mathrm{x}$ and $\mathrm{y}$ axis. The angular velocity value was generally reduced from the control group to patients. Therefore, the knee and the ankle joints suffered the most angular velocity changes. The main reason for significant angular velocity reduction in knee and ankle joints was due to the foot drop that actually disrupts the order of the cycle gate. Patient $s$ inability in dorsi-flexion and plantar-flexion of the ankle joint during walking had a significant effect on the angular velocity in the joint. The y axis endured the most significant decrease in angular velocity of the heel joint. The hip joint lacked any significant variation and regarded as the least changeable joint under the influence of the disease. While most of the changes seen in y and $\mathrm{x}$ axes, no significant changes observed in the $\mathrm{z}$ axis from the healthy to the patient group. Musculoskeletal analysis of the lower extremities in the stance phase, is a clear indication of significant changes in the $\mathrm{x}$ and $\mathrm{y}$ axes.

The kinetics analysis suggested a significant increase in the $b_{1}$ coefficient from the control group to the patient group. In the stance phase of control group, the stages of heel contact and Toe off were performed regularly. In CMT patients the maximum force of the soles was not generated in the heels and transmitted forward, due to sudden pounding of the soles to the ground. This phenomenon could justify the significant increase of the $b_{1}$ coefficient.

Subtractive changes of $c_{1}$ coefficient in the patient group indicated a significant reduction in the data variations and the uncontrolled distribution of forces in soles of CMT patients. The lack of regular Heel contact and Toe off in CMT patients could limit the range of sole forces compared to the control group. The maximum force variation was observed along the $\mathrm{x}$ axis, which was related to the application of sole forces to the front and back directions. In fact, the ankle relaxation can be a limiting factor for the force exertion in $\mathrm{x}$ direction.

Unfortunately, the limited access to CMT patients prevented us from creating a larger statistical population. Though, the results were somehow comparable to the achievements of the relevant previous studies, a larger statistical population could provide more reliable results. In addition, due to the limited number of available patients, the high age differences between the diseased and control groups could not be neglected.

\section{Conclusion}

Due to the ankle range of motion in CMT disease, the patients had limited movements in the ankle flexion and extension. These limitations had the greatest impact in $\mathrm{x}$ and $\mathrm{y}$ directions. Angular velocity was significantly reduced under the influence of disease. The wide range of foot forces in the control group was not observed in patients. Also, the maximum foot force of the patients was measured in the fore-heel when taking a new step. Therefore, CMT motion models were extracted to establish objective diagnostic criteria.

\section{Conflict of Interest}

The authors report neither financial support nor conflict of interest.

\section{Clinical Relevance}

1) The kinematics and kinetics characteristics of Charcot Marie Tooth patients were investigated using motion analysis tests.

2) The CMT motion model parameters were extracted and compared with a control group.

3) CMT motion models were extracted to establish objective diagnostic criteria. 


\section{References}

[1] Krajewski, K. M., et al. (2000). Neurological dysfunction and axonal degeneration in Charcot-Marie-Tooth disease type 1A. Brain, 123(Pt 7): 1516-27.

[2] Szigeti, K., Lupski, J. R. (2009). Charcot-Marie-Tooth disease. European Journal of Human Genetics, 17: 703-710.

[3] Jani-Acsadi, A., et al. (2008). Charcot-Marie-Tooth Neuropathies: Diagnosis and Management. Neuromuscular Disorders; M.D.Semin Neurol, 28: 185-194.

[4] Yiu Eppie, M., et al. (2008). Neurophysiologic abnormalities in children with Charcot-Marie-Tooth disease type 1A. Journal of the Peripheral Nervous System, 13(3): 236-41.

[5] Gemignani, F., et al. (1999). Charcot-Marie-Tooth disease type 2 with restless legs syndrome. Neurology, 52: 1064-1066.

[6] Mostacciuolo, M. L., et al. (2001). Charcot-Marie-Tooth disease type I and related demyelinating neuropathies: mutation analysis in a large cohort of Italian families. Human Mutation, 18: 32-41.

[7] Berciano, J., et al. (2011). Charcot-Marie-Tooth disease: a review with emphasis on the pathophysiology of pes cavus. Revista Española de Cirugía Ortopédica y Traumatología (English Edition), 55(2): 140-150.

[8] Le, T., Bhushan, V. (2001). 201 First Aid for the USMLE Step 14. McGraw-Hill Education, USA.

[9] Soykan, I., McCallum, R. W. (1997). Gastrointestinal involvement in neurologic disorders: Stiff-man and Charcot-Marie-Tooth syndromes. The American Journal of the Medical Sciences, 313(1): 70-73.

[10] Fernando, M., et al. (2013). Biomechanical characteristics of peripheral diabetic neuropathy: A systematic review and meta-analysis of findings from the gait cycle, muscle activity and dynamic barefoot plantar pressure. Clinical Biomechanics, 28: 831-845.

[11] Huang, C. K., et al. (2019). An altered spatiotemporal gait adjustment during a virtual obstacle crossing task in patients with diabetic peripheral neuropathy. Journal of Diabetes and its Complications, 33: 182-188.

[12] Fernando, M., et al. (2013). Biomechanical characteristics of peripheral diabetic neuropathy: A systematic review and meta-analysis of findings from the gait cycle, muscle activity and dynamic barefoot plantar pressure. Clinical Biomechanics, 28: 831-845.

[13] Leardini, A., et al. (2007). Rear-foot, mid-foot and forefoot motion during the stance phase of gait. Gait Posture, 25: $453-462$.

[14] Manal, K., Stanhope, S. J. (2004). A novel method for displaying gait and clinical movement analysis data. Gait Posture, 20: 222-226.

[15] De Ridder, R., et al. (2013). Gait kinematics of subjects with ankle instability using a multisegmented foot model. Medicine \& Science in Sports \& Exercise, 45: 2129-36.

[16] Fernando, M., et al. (2013). Biomechanical characteristics of peripheral diabetic neuropathy: A systematic review and meta-analysis of findings from the gait cycle, muscle activity and dynamic barefoot plantar pressure. Clinical Biomechanics, 28: 831-845.

[17] Rezende, A., et al. (2013). Muscle strength and ankle mobility for the gait parameters in diabetic neuropathies. Clinical Biomechanics, 23: 17-21.

[18] Deschamps, K., et al. (2013). Comparison of foot segmental mobility and coupling during gait between patients with diabetes mellitus with and without neuropathy and adults without diabetes. Clinical Biomechanics, 28: 813-819.

[19] Ashofteh Yazdi, A., Esteki, A., and Dehghan, M. M. (2019). Determination of an average quasi-linear viscoelastic model for the mechanical behavior of rat cervix. Proceedings of the Institution of Mechanical Engineers, Part L: Journal of Materials: Design and Applications, 233(5): 924-929.

[20] Yazdi, A. A., Esteki, A., Dehghan, M. M., and Ghomsheh, F. T. (2016). Characterization of viscoelastic behavior of rat cervix in the last trimester of pregnancy. Biomed Res, 27(4): 1194-1200.

[21] Yazdi, A. A., Melchor, J., Torres, J., Faris, I., Callejas, A., Gonzalez-Andrades, M., and Rus, G. (2020). Characterization of non-linear mechanical behavior of the cornea. Scientific Reports, 10(1): 1-10. 